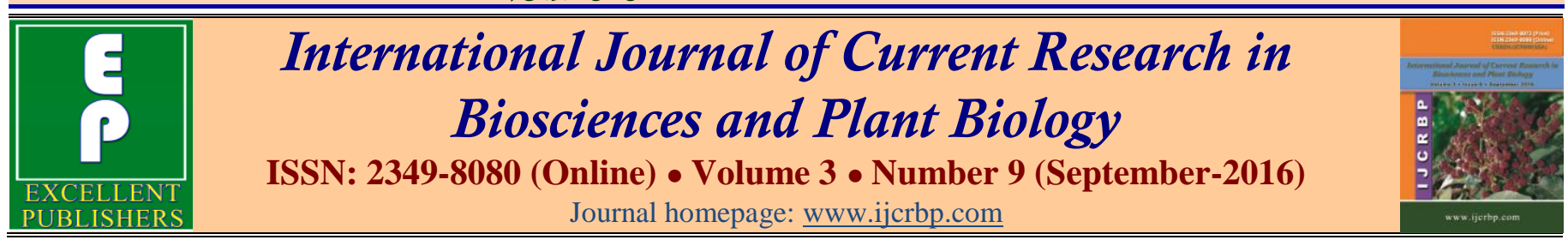

\title{
Per Se Performance of Chilli Genotypes and Hybrids Across Four Environments for Yield, Horticultural and Quality Traits
}

\author{
Munish Sharma $^{1 *}$ and Akhilesh Sharma ${ }^{2}$ \\ ${ }^{1}$ Krishi Vigyan Kendra Mohali, ${ }^{2}$ Department of Vegetable Science and Floriculture, CSK Himachal Pradesh Agricultural \\ University, Palampur, 176 o62, India
}

*Corresponding author.

\begin{abstract}
A bstract
An experiment was conducted for two consecutive years at two different locations to identify the best genotypes based on per se performance in chilli for yield and component traits along with horticultural and quality traits. The thirty three $\mathrm{F}_{1} \mathrm{~s}$, fourteen parents and standard check ' $\mathrm{CH}-1$ ' were grown in a Completely Randomized Block Design with three replications at Palampur and Bajaura for two consecutive summer-rainy seasons during 2010 and 2011. Existence of sufficient genetic variability among treatments was evident from the analysis of variance for all the characters in pooled over environments. A wide variation was observed in the mean performance of 14 parents and their 33 crosses derived from these parents for different traits pooled over environments. Cross 'LCA $436 \times$ Pant C 1' recorded the highest marketable fruit yield $(615.23 \mathrm{~g})$ and dry fruit yield $(70.59 \mathrm{~g})$ which was 68.37 per cent and 47.27 per cent higher over the standard check ' $\mathrm{CH}-1$ ', respectively in pooled over environments. This cross also produced maximum fresh and dry yield /plant in the respective environments over the years.
\end{abstract}

\author{
Article Info \\ Accepted: 11 August 2016 \\ Available Online: 06 September 2016 \\ Ke ywords \\ Capsicum annuum var. annuum $\mathrm{L}$. \\ Per se performance \\ Randomized block design
}

\section{Introduction}

Chilli (Capsicum annuum L.) is a member of the Solanaceae family, originated from South and Central America. Chilli is an indispensable spice due to its pungency, taste, appealing colour and flavor and has its unique place in the diet as a vegetable cum spice crop. India is the largest producer, consumer and exporter of chilli in the world (Janaki et al., 2015). Immature chilli fruits contain phytonutrients, ascorbic acid, caretenoids and rutin which are valued for pharmaceutical needs. Chillies have two important qualities; biting pungency and attractive red colour attributed to capsaicin and capsanthin, respectively. Capsaicin, a crystalline acrid volatile alkaloid present in the placenta of fruit, carries diverse prophylactic and therapeutic uses in allopathic and ayurvedic medicines. Red coloured pigment is used as a natural colour additive in food, drugs and cosmetics. These pigments are also rich in bioflavonoids, which are powerful antioxidants and inhibit the progression of chronic diseases such as muscular degeneration, cardiovascular diseases and cancer. Oleoresin extracted from dried and ground chillies is the total flavour extract which has gained industrial importance through its utilization in processed products and pharmaceutical formulations. Oleoresin is gaining more importance especially from export point of view as it offers uniform quality, longer shelf-life, freedom from micro-organisms and lesser freight charges. In the present study per se performance of chilli genotypes and hybrids in four diverse environments was studied. 


\section{Materials and methods}

The thirty three $\mathrm{F}_{1} \mathrm{~s}$, fourteen parents and standard check ' $\mathrm{CH}-1$ ' were grown in a Completely Randomized Block Design with three replications at Palampur and Bajaura for two consecutive summerrainy seasons during 2010 and 2011 . Seeds were sown in nursery beds of size $3 \mathrm{~m} \times 1 \mathrm{~m}$ on $2^{\text {nd }}$ and $6^{\text {th }}$ March at Palampur and Bajaura during 2010, respectively, whereas, seed sowings in the respective environments were carried out on $7^{\text {th }}$ and $10^{\text {th }}$ April during 2011. Twelve plants of $10-15 \mathrm{~cm}$ height were transplanted in the field on $20^{\text {th }}$ and $22^{\text {nd }}$ April during 2010 and $4^{\text {th }}$ and $10^{\text {th }}$ May during 2011at Palampur and Bajaura, respectively, with inter and intra-row spacing of $45 \mathrm{~cm}$ each.

The experimental fields in the respective environments were ploughed using a 3-disc tractor and twice using a 7-disc tractor followed by power tiller. The recommended farmyard manure @ 20 tonnes/hectare was mixed in the soil at time of field preparation. The fertilizers were applied @ 75: 60: $60 \mathrm{~kg} \mathrm{~N}, \mathrm{P}_{2} \mathrm{O}_{5}$ and $\mathrm{K}_{2} \mathrm{O} /$ hectare with half of recommended $\mathrm{N}$, full $\mathrm{P}$ and $\mathrm{K}$ at planting time and remaining $\mathrm{N}$ in two equal splits at one month interval after planting. Irrigation was provided one week prior to planting and immediately after transplanting for proper establishment of plants in the soil and thereafter, at ten days interval prior to the onset of monsoon. Five hand weeding were carried out at monthly interval to keep the field weed free. Drainage was also provided to keep the fields free from stagnation of water during rainy season. The harvestings were carried out manually. The experimental material for the present study comprised of $F_{1}$ population of 33 crosses which were developed by crossing 11 lines of chilli, viz., 'Jawahar Mirch 283', 'Chilli Sonal' ,PAU Selection Long', 'Arka Lohit' 'LCA 436', 'Pusa Jwala', 'Pusa Sadabahar', 'Kashmir Long', 'Selection 352', 'LCA 443' and 'LCA 206' and with three testers, viz., 'Pant C 1', 'Anugraha' and 'Surajmukhi'.

Hybrid ' $\mathrm{CH}-1$ ' was used as a standard check. The observations were recorded on yield, horticultural and quality traits viz., days to $50 \%$ flowering, days to first harvest, primary branches/plant fruit length $(\mathrm{cm})$, fruit girth $(\mathrm{cm})$, average fruit weight $(\mathrm{g})$, marketable fruits/plant, marketable fruit yield/plant $(\mathrm{g})$, harvest duration (days), plant height $(\mathrm{cm})$, average dry fruit weight $(\mathrm{g})$, dry fruit yield/plant (g), ascorbic acid (mg/100g), capsaicin content (\%), capsanthin (ASTA units), oleoresin (ASTA units). Ascorbic acid content in chilli was estimated by '2,6-dichlorophenolindophenol Visual Titration Method' as described by Ranganna (1979). The capsaicin content in the fruits was determined by calorimetric method using FolinCiocalteau reagent described by Bajaj (1980). Capsanthin and oleoresin were determined as per procedure given by A.O.A.C. (1980). The statistical analysis is done as per Gomez and Gomez (1983).

\section{Results and discussion}

Existence of sufficient genetic variability among treatments was evident from the analysis of variance (Table 1) for all the characters namely, days to $50 \%$ flowering, days to first harvest, primary branches/plant, fruit length, fruit girth, average fruit weight, marketable fruits/plant, marketable fruit yield/plant, plant height, harvest duration, average dry fruit weight, dry fruit yield/plant, ascorbic acid, capsaicin content, capsanthin and oleoresin in both the environments at Palampur and Bajaura during both the years and pooled over environments. The pooled analysis of variance over the four environments also exhibited significant $G \times E$ interaction for all the characters studied indicating that performance of genotypes/crosses was greatly influenced by environments.

A wide variation was observed in the mean performance of 14 parents and their 33 crosses derived from these parents for different traits pooled over environments. High yield is the basic objective of all crop improvement programs. For marketable fruit yield/plant, 16 and 14 cross combinations in the respective years at Palampur, 19 crosses each during both the years at Bajaura and 18 crosses in pooled over environments significantly surpassed the commercial hybrid ' $\mathrm{CH}-1$ ' (Table 2). Cross 'LCA 436 $\times$ Pant C 1' recorded the highest marketable fruit yield $(615.23 \mathrm{~g})$ and dry fruit yield $(70.59 \mathrm{~g})$ which was 68.37 per cent and 47.27 per cent higher over the standard check ' $\mathrm{CH}-1$ ', respectively in pooled over environments. This cross also produced maximum fresh and dry yield /plant in the respective environments over the years except at Palampur during 2010 where it secured second position with respect to fresh fruit yield and third position for dry fruit yield. 'Jawahar Mirch $283 \times$ Anugraha' significantly outperformed all the crosses and ranked first during 2010 and second in 2011 at Palampur for marketable fruit yield. 
Table 1. Pooled over environment analysis of variance for various traits in chilli.

\begin{tabular}{|c|c|c|c|c|c|}
\hline $\begin{array}{l}\text { Source of variation/Traits } \\
\text { df }\end{array}$ & $\begin{array}{l}\text { Locations } \\
3\end{array}$ & $\begin{array}{l}\text { Replications } \\
8 \\
\end{array}$ & $\begin{array}{l}\text { Treatments } \\
47\end{array}$ & $\begin{array}{l}\text { Location } x \\
\text { Treatments } \\
141\end{array}$ & $\begin{array}{l}\text { Pooled } \\
\text { Error } \\
\mathbf{3 7 6} \\
\end{array}$ \\
\hline Days to $50 \%$ flowering & $912.29 *$ & $5.65 *$ & $302.95 *$ & $21.62 *$ & 1.64 \\
\hline Primary branches/plant & $26.87 *$ & $1.28 *$ & $4.03 *$ & $1.10 *$ & 0.19 \\
\hline Fruit length $(\mathrm{cm})$ & $13.92 *$ & 0.08 & $13.24 *$ & $2.05 *$ & 0.06 \\
\hline Average fruit weight (g) & $0.24 *$ & $0.009 *$ & $16.83 *$ & $0.07 *$ & 0.002 \\
\hline Marketable fruits/ plant & $13639.33 *$ & $158.97 *$ & $19628.16^{*}$ & $1783.92 *$ & 33.93 \\
\hline Marketable fruit yield/plant (g) & $191597.30^{*}$ & $1683.83 *$ & $146673.90 *$ & $13744.32 *$ & 332.58 \\
\hline Harvest duration (days) & $38.17 *$ & $5.49 *$ & $377.24 *$ & $2.66^{*}$ & 0.69 \\
\hline Plant height $(\mathrm{cm})$ & $4386.42 *$ & $22.15^{*}$ & $522.42 *$ & $100.58 *$ & 8.87 \\
\hline Capsaicin content (\%) & $0.02 *$ & 0.001 & $0.31 *$ & $0.003^{*}$ & 0.000 \\
\hline Capsanthin (ASTA units) & $55.67 *$ & $20.42 *$ & $1684.98 *$ & $32.50 *$ & 3.04 \\
\hline Oleoresin (ASTA units) & $250.17 *$ & $33.54 *$ & $2119.98 *$ & $35.70 *$ & 2.77 \\
\hline
\end{tabular}

* Significant at $p \leq 0.05$

On the other hand, 'Arka Lohit $\times$ Surajmukhi' was the top ranking cross combination for dry fruit yield during 2010 and also secured second position in 2011 at Palampur and pooled over environments. In addition to these top performing crosses, 'LCA $436 \times$ Anugraha, 'PAU Selection Long $\times$ Surajmukhi', 'Arka Lohit $\times$ Surajmukhi' and 'LCA $443 \times$ Surajmukhi' also significantly outyielded ' $\mathrm{CH}-1$ ' (standard check) with consistency by retaining positions among top ten crosses in both the years at respective locations and also pooled over environments for fresh fruit yield/plant. Moreover, 'Jawahar Mirch $283 \times$ Surajmukhi' and 'Pusa Jwala $\times$ Surajmukhi' at Palampur and 'Chilli Sonal $\times$ Surajmukhi' at Bajaura during both the years were the other promising crosses with high fresh fruit yield.

For dry fruit yield/plant, 'PAU Selection Long $\times$ Surajmukhi' and 'Jawahar Mirch $283 \times$ Anugraha' were the other promising crosses with consistent performance among top ten over the years and environments which was similar to their performance for fresh fruit yield. Further, cross combinations 'Arka Lohit $\times$ Anugraha', 'LCA $443 \times$ Surajmukhi' and 'Kashmir Long $\times$ Surajmukhi' at Palampur and 'Chilli Sonal $\times$ Surajmukhi', 'Selection $352 \times$ Surajmukhi' and 'Jawahar Mirch $283 \times$ Pant C 1' at Bajaura also outperformed the standard check ' $\mathrm{CH}-1$ ' during both the years by securing position among top ten crosses for dry fruit yield/plant.
The superior performance of all these crosses for both fresh and dry fruit yield /plant was mainly attributed to fruit length, marketable fruits/plant, harvest duration and plant height which was evident from the significant better performance for these traits compared to check ' $\mathrm{CH}-1$ '. However, at Palampur, the performance of 'Arka Lohit $\times$ Surajmukhi' during 2010 and 'PAU Selection Long $\times$ Surajmukhi', 'Arka Lohit $\times$ Surajmukhi', 'Kashmir Long $\times$ Surajmukhi' and 'LCA $443 \times$ Surajmukhi' during 2011 for plant height and 'Arka Lohit $\times$ Anugraha' for marketable fruits/plant during 2010 was at par with ' $\mathrm{CH}-1$ '. Similarly, 'Selection $352 \times$ Surajmukhi' for fruit length during both the years and 'Jawahar Mirch $283 \times$ Pant C 1' for plant height during 2010 at Bajaura and 'Jawahar Mirch $283 \times$ Anugraha' for harvest duration over the years and environments performed at par with ' $\mathrm{CH}-1$ '. Similar findings have been reported by Datta and Jana (2011) and Puttapalli and Bhoyar (2016) in their studies.

For quality traits, among these promising crosses, 'PAU Selection Long $\times$ Surajmukhi' for ascorbic acid, 'LCA $436 \times$ Pant C 1', 'LCA $436 \times$ Anugraha' and 'LCA 443 $\times$ Surajmukhi' for capsanthin also outperformed significantly the standard check ' $\mathrm{CH}-1$ ', while the performance of 'Arka Lohit $\times$ Surajmukhi' for capsaicin was similar to ' $\mathrm{CH}-1$ ' on the basis of pooled over environments. 
Table 2. Mean values of fourteen parents, thirty three $\mathrm{F}_{1} \mathrm{~s}$ and one check for the characters studied at pooled over environments.

\begin{tabular}{|c|c|c|c|c|c|c|c|c|c|c|c|c|c|c|c|c|c|}
\hline$\underset{i}{\dot{0}}$ & 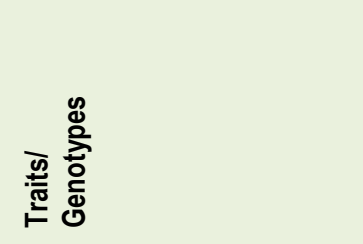 & 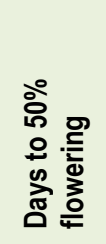 & 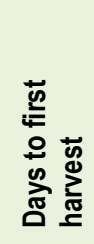 & 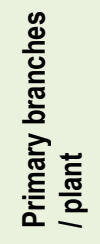 & 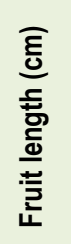 & 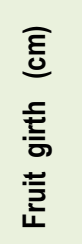 & 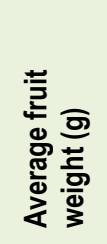 & 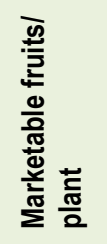 & 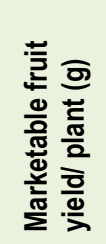 & 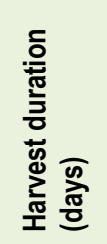 & 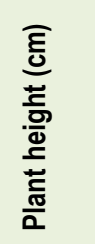 & 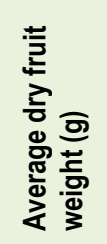 & 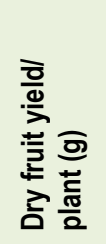 & 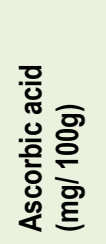 & 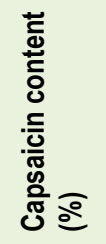 & 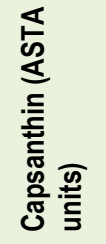 & 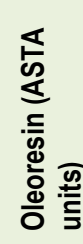 \\
\hline 1 & Jawahar Mirch 283 & 51.67 & 73.67 & 5.90 & 8.31 & 1.00 & 3.27 & 80.87 & 264.58 & 51.17 & 65.79 & 0.73 & 28.88 & 94.38 & 0.50 & 74.83 & 54.17 \\
\hline 2 & Chilli Sonal & 47.58 & 64.17 & 6.63 & 7.41 & 0.91 & 2.21 & 91.10 & 199.50 & 41.42 & 64.82 & 0.78 & 30.38 & 93.38 & 0.28 & 75.50 & 30.63 \\
\hline 3 & PAU Sel Long & 53.67 & 75.67 & 6.50 & 7.27 & 0.94 & 3.43 & 65.04 & 223.00 & 52.17 & 64.29 & 1.28 & 31.53 & 106.96 & 0.50 & 79.70 & 53.33 \\
\hline 4 & Arka Lohit & 49.50 & 66.00 & 6.58 & 7.95 & 1.28 & 5.06 & 52.59 & 265.82 & 52.67 & 64.32 & 0.67 & 21.29 & 117.29 & 0.52 & 70.92 & 65.86 \\
\hline 5 & LCA 436 & 53.58 & 71.25 & 6.37 & 8.76 & 1.33 & 6.32 & 38.76 & 245.45 & 53.08 & 70.95 & 1.99 & 33.54 & 100.88 & 0.52 & 77.06 & 54.53 \\
\hline 6 & Pusa Jwala & 48.42 & 70.00 & 6.10 & 9.12 & 0.82 & 2.34 & 112.09 & 261.75 & 47.00 & 54.30 & 0.85 & 33.10 & 104.46 & 0.87 & 78.05 & 82.00 \\
\hline 7 & Pusa Sadabahar & 60.92 & 81.83 & 6.06 & 5.29 & 0.97 & 3.60 & 51.55 & 185.92 & 60.67 & 60.08 & 0.67 & 26.83 & 104.38 & 0.82 & 94.42 & 76.63 \\
\hline 8 & Kashmir Long & 42.25 & 63.25 & 5.70 & 8.64 & 1.13 & 5.15 & 53.16 & 274.11 & 52.08 & 67.38 & 0.68 & 31.18 & 108.08 & 0.35 & 92.04 & 53.25 \\
\hline 9 & Sel 352 & 51.25 & 70.50 & 6.40 & 6.23 & 1.21 & 4.22 & 54.57 & 230.58 & 53.25 & 67.84 & 1.63 & 24.92 & 101.55 & 0.45 & 75.17 & 51.25 \\
\hline 10 & LCA 443 & 61.00 & 80.42 & 5.58 & 7.08 & 1.44 & 5.09 & 56.04 & 282.13 & 52.58 & 61.65 & 2.25 & 28.93 & 106.92 & 0.45 & 74.37 & 49.71 \\
\hline 11 & LCA 206 & 54.83 & 80.92 & 6.50 & 8.48 & 0.84 & 2.03 & 88.52 & 179.41 & 51.83 & 76.63 & 0.92 & 23.63 & 100.50 & 0.54 & 73.90 & 59.01 \\
\hline 12 & Pant C 1 & 59.63 & 82.67 & 6.18 & 5.13 & 1.03 & 2.07 & 88.30 & 183.17 & 62.58 & 55.23 & 0.77 & 27.83 & 129.13 & 0.64 & 78.18 & 61.38 \\
\hline 13 & Anugraha & 50.75 & 68.50 & 7.67 & 8.12 & 0.74 & 2.32 & 104.26 & 241.59 & 63.42 & 61.30 & 0.64 & 30.18 & 102.54 & 0.48 & 88.67 & 61.75 \\
\hline 14 & Surajmukhi & 61.88 & 79.83 & 7.95 & 6.52 & 0.97 & 4.57 & 62.21 & 284.32 & 62.33 & 65.45 & 0.65 & 33.13 & 112.04 & 0.89 & 101.46 & 65.81 \\
\hline 15 & Jawahar Mirch 283×Pant C 1 & 45.42 & 70.83 & 6.68 & 7.10 & 1.09 & 3.79 & 119.05 & 450.26 & 47.00 & 63.32 & 0.83 & 57.88 & 110.63 & 0.38 & 101.10 & 44.00 \\
\hline 16 & $\begin{array}{l}\text { Jawahar Mirch } 283 \times \\
\text { Anugraha }\end{array}$ & 46.17 & 67.92 & 7.47 & 8.66 & 1.07 & 3.09 & 176.85 & 543.35 & 51.42 & 80.23 & 0.72 & 62.05 & 97.88 & 0.43 & 111.46 & 50.67 \\
\hline 17 & $\begin{array}{l}\text { Jawahar Mirch } 283 \text { × } \\
\text { Surajmukhi }\end{array}$ & 46.67 & 73.00 & 6.85 & 8.58 & 1.09 & 2.91 & 153.49 & 443.73 & 54.67 & 81.01 & 1.01 & 54.39 & 103.13 & 0.53 & 106.97 & 50.92 \\
\hline 18 & Chilli Sonal $\times$ Pant C 1 & 43.50 & 63.00 & 7.00 & 6.22 & 0.99 & 2.39 & 112.55 & 268.64 & 47.67 & 67.52 & 1.08 & 34.93 & 115.96 & 0.31 & 76.68 & 44.21 \\
\hline 19 & Chilli Sonal $\times$ Anugraha & 44.75 & 64.67 & 7.22 & 6.76 & 0.94 & 3.16 & 94.04 & 296.49 & 42.33 & 71.09 & 0.81 & 32.00 & 113.04 & 0.34 & 75.53 & 35.92 \\
\hline 20 & Chilli Sonal $\times$ Surajmukhi & 43.50 & 75.58 & 6.85 & 7.65 & 0.89 & 2.31 & 206.18 & 472.82 & 58.50 & 77.95 & 1.04 & 57.76 & 99.63 & 0.46 & 94.79 & 54.96 \\
\hline 21 & PAU Sel Long $\times$ Pant C 1 & 47.42 & 72.08 & 6.47 & 7.98 & 0.84 & 2.50 & 152.80 & 381.82 & 53.17 & 67.62 & 0.85 & 48.13 & 104.29 & 0.49 & 92.48 & 63.71 \\
\hline 22 & PAU Sel Long $\times$ Anugraha & 48.17 & 74.33 & 6.88 & 7.71 & 0.83 & 2.69 & 151.07 & 406.38 & 57.50 & 65.03 & 0.93 & 48.88 & 116.75 & 0.58 & 94.54 & 63.58 \\
\hline 23 & PAU Sel Long $\times$ Surajmukhi & 46.17 & 73.33 & 7.02 & 8.16 & 0.94 & 3.08 & 170.57 & 524.04 & 61.42 & 77.22 & 1.40 & 63.28 & 129.63 & 0.64 & 110.29 & 67.79 \\
\hline 24 & Arka Lohit x Pant C 1 & 47.92 & 73.83 & 6.97 & 7.30 & 0.87 & 3.44 & 114.29 & 392.62 & 55.75 & 65.79 & 1.59 & 50.83 & 119.02 & 0.69 & 99.92 & 72.38 \\
\hline 25 & Arka Lohit × Anugraha & 47.92 & 72.08 & 6.17 & 7.44 & 1.04 & 6.91 & 57.71 & 399.38 & 60.08 & 69.24 & 1.24 & 56.36 & 122.08 & 0.70 & 90.75 & 81.96 \\
\hline
\end{tabular}




\begin{tabular}{|c|c|c|c|c|c|c|c|c|c|c|c|c|c|c|c|c|c|}
\hline$\dot{\sum}_{\dot{0}}^{\dot{0}}$ & 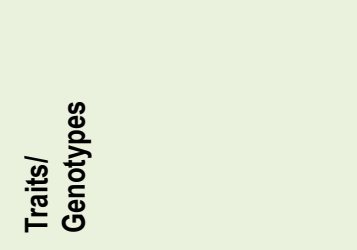 & 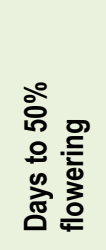 & 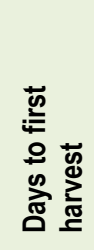 & 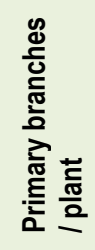 & 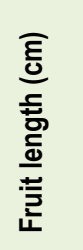 & 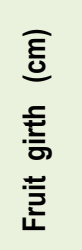 & 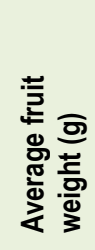 & 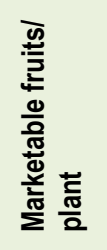 & 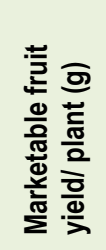 & 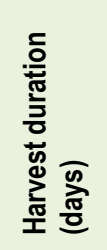 & 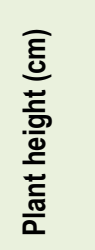 & 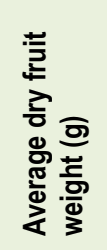 & 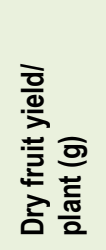 & 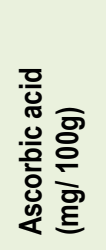 & 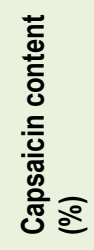 & 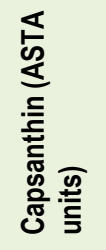 & 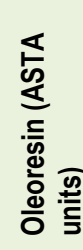 \\
\hline 26 & Arka Lohit $\times$ Surajmukhi & 46.42 & 66.58 & 7.48 & 7.35 & 1.04 & 5.08 & 102.65 & 520.96 & 56.67 & 71.44 & 1.07 & 64.37 & 95.83 & 0.76 & 87.72 & 73.42 \\
\hline 27 & LCA $436 \times$ Pant C 1 & 43.92 & 67.50 & 6.58 & 7.78 & 1.16 & 4.02 & 153.15 & 615.23 & 59.67 & 70.74 & 1.49 & 70.59 & 95.79 & 0.49 & 104.33 & 56.29 \\
\hline 28 & LCA $436 \times$ Anugraha & 43.92 & 66.42 & 6.62 & 9.19 & 0.97 & 3.58 & 150.32 & 536.98 & 62.25 & 67.57 & 1.31 & 58.87 & 103.42 & 0.40 & 101.72 & 37.96 \\
\hline 29 & LCA $436 \times$ Surajmukhi & 47.67 & 66.83 & 7.20 & 8.11 & 1.05 & 4.49 & 96.03 & 430.67 & 56.75 & 70.35 & 1.71 & 54.38 & 114.88 & 0.46 & 107.58 & 52.42 \\
\hline 30 & Pusa Jwala $\times$ Pant C 1 & 46.33 & 64.83 & 6.80 & 7.16 & 0.94 & 2.38 & 129.43 & 305.16 & 51.58 & 54.96 & 1.00 & 38.89 & 95.08 & 0.87 & 80.10 & 84.96 \\
\hline 31 & Pusa Jwala $\times$ Anugraha & 45.33 & 63.00 & 7.27 & 8.22 & 0.84 & 2.71 & 130.28 & 351.01 & 50.92 & 57.34 & 1.01 & 44.04 & 112.50 & 0.77 & 95.25 & 77.96 \\
\hline 32 & Pusa Jwala × Surajmukhi & 44.83 & 62.33 & 6.70 & 8.14 & 0.78 & 3.75 & 123.63 & 464.19 & 50.50 & 65.32 & 1.01 & 55.07 & 100.88 & 0.84 & 86.83 & 82.67 \\
\hline 33 & Pusa Sadabahar ×Pant C 1 & 50.50 & 71.92 & 7.05 & 7.22 & 0.86 & 2.82 & 87.99 & 247.45 & 57.67 & 68.83 & 1.35 & 31.18 & 117.38 & 0.71 & 75.96 & 71.75 \\
\hline 34 & Pusa Sadabahar $\times$ Anugraha & 49.17 & 77.83 & 7.50 & 5.99 & 0.86 & 2.55 & 103.59 & 264.44 & 62.83 & 66.71 & 0.66 & 33.87 & 124.33 & 0.70 & 76.73 & 77.83 \\
\hline 35 & $\begin{array}{l}\text { Pusa Sadabahar x } \\
\text { Surajmukhi }\end{array}$ & 47.00 & 71.75 & 7.43 & 7.91 & 1.06 & 2.10 & 209.20 & 433.58 & 64.08 & 77.07 & 0.65 & 49.43 & 128.92 & 0.66 & 87.19 & 79.77 \\
\hline 36 & Kashmir Long $\times$ Pant C 1 & 41.58 & 59.75 & 5.35 & 8.02 & 1.00 & 3.92 & 97.36 & 381.86 & 46.42 & 64.93 & 1.28 & 52.23 & 119.38 & 0.83 & 103.63 & 77.88 \\
\hline 37 & Kashmir Long $\times$ Anugraha & 42.00 & 59.67 & 5.27 & 9.76 & 0.93 & 3.18 & 111.62 & 354.70 & 47.75 & 69.04 & 0.74 & 44.33 & 130.46 & 0.51 & 84.08 & 55.67 \\
\hline 38 & Kashmir Long $\times$ Surajmukhi & 42.67 & 62.42 & 6.68 & 8.34 & 0.96 & 4.19 & 104.24 & 440.63 & 51.33 & 65.86 & 0.67 & 52.35 & 123.96 & 0.62 & 85.01 & 63.21 \\
\hline 39 & Sel $352 \times$ Pant C 1 & 50.92 & 72.67 & 6.48 & 6.08 & 1.12 & 4.16 & 93.56 & 385.71 & 50.33 & 71.41 & 1.05 & 52.65 & 99.71 & 0.69 & 86.17 & 70.21 \\
\hline 40 & Sel $352 \times$ Anugraha & 44.50 & 61.75 & 6.48 & 7.30 & 0.96 & 4.66 & 86.95 & 404.98 & 52.50 & 66.28 & 1.20 & 53.88 & 93.83 & 0.69 & 87.06 & 67.75 \\
\hline 41 & Sel $352 \times$ Surajmukhi & 48.83 & 65.75 & 6.87 & 6.41 & 1.02 & 3.72 & 115.92 & 431.10 & 50.92 & 80.42 & 1.33 & 58.27 & 104.79 & 0.76 & 109.17 & 74.92 \\
\hline 42 & LCA $443 \times$ Pant C 1 & 44.92 & 70.17 & 6.71 & 7.36 & 1.19 & 4.38 & 89.87 & 393.17 & 47.58 & 75.97 & 1.06 & 44.61 & 120.25 & 0.67 & 83.63 & 72.58 \\
\hline 43 & LCA $443 \times$ Anugraha & 49.08 & 70.42 & 7.05 & 8.83 & 1.09 & 4.57 & 87.62 & 400.88 & 47.08 & 70.78 & 1.29 & 52.43 & 103.13 & 0.61 & 89.88 & 60.46 \\
\hline 44 & LCA $443 \times$ Surajmukhi & 46.08 & 71.50 & 6.81 & 7.58 & 1.23 & 5.10 & 103.89 & 529.68 & 55.00 & 75.48 & 1.48 & 60.54 & 111.13 & 0.65 & 110.67 & 71.13 \\
\hline 45 & LCA $206 \times$ Pant C 1 & 53.83 & 71.42 & 6.28 & 7.95 & 0.92 & 2.96 & 77.48 & 229.25 & 50.33 & 68.15 & 0.94 & 30.71 & 93.42 & 0.57 & 79.89 & 60.04 \\
\hline 46 & LCA $206 \times$ Anugraha & 46.58 & 69.00 & 6.72 & 10.14 & 0.84 & 1.81 & 173.48 & 307.40 & 52.42 & 70.47 & 1.16 & 37.97 & 107.83 & 0.68 & 81.42 & 67.54 \\
\hline 47 & LCA $206 \times$ Surajmukhi & 55.50 & 70.17 & 6.93 & 7.28 & 0.83 & 2.14 & 130.74 & 281.08 & 59.92 & 77.38 & 1.03 & 39.45 & 121.96 & 0.73 & 77.25 & 71.75 \\
\hline \multirow[t]{4}{*}{48} & $\mathrm{CH}-1$ & 48.08 & 66.75 & 5.85 & 6.58 & 1.24 & 4.97 & 73.47 & 365.40 & 51.75 & 60.18 & 1.71 & 47.93 & 110.17 & 0.79 & 88.88 & 83.46 \\
\hline & Mean & 48.63 & 70.00 & 6.66 & 7.64 & 1.00 & 3.57 & 107.09 & 353.67 & 53.79 & 68.18 & 1.09 & 43.75 & 109.24 & 0.60 & 88.73 & 63.35 \\
\hline & CV $(\%)$ & 2.63 & 1.90 & 6.58 & 3.34 & 3.58 & 1.40 & 5.44 & 5.16 & 1.55 & 4.37 & 8.32 & 6.61 & 2.00 & 2.80 & 1.96 & 2.63 \\
\hline & $C D$ at $P \leq 0.05$ & 2.08 & 2.16 & 0.71 & 0.41 & 0.06 & 0.08 & 9.44 & 29.56 & 1.35 & 4.83 & 0.15 & 4.69 & 3.55 & 0.03 & 2.82 & 2.70 \\
\hline
\end{tabular}




\section{Conflict of interest statement}

Authors declare that they have no conflict of interest.

\section{References}

AOAC., 1980. Official Methods of Analysis. Association of Official Analytical Chemists (Ed.: William Horwitz). Benzamin Franklin Station, Washington, D.C.

Bajaj, K.L., 1980. Calorimetric determination of capsaicin in capsicum fruits. J. Assoc. Offi. Anal. Chemist. 63(6), $1314-1316$

Datta, S., Jana, J. C., 2011. Performance of chilli (Capsicum annum) genotypes under Terai agroclimatic region of West Bengal. Ind. J. Agric. Sci. 81(6), 567-570.

Gomez, K. A., Gomez, A. A., 1983. Statistical Procedures for Agricultural Research. John Wiley and Sons. 690p.

Janaki, M., Venkata Ramana, C., Naram Naidu, L., Paratpara Rao, M., 2015. Performance of chilli (Capsicum annuиm L.) genotypes for yield and yield attributing traits. Plant Arch. 15(2), 661-666.

Raja Sekhar, P., Bhoyar, S. M., 2016. Performance of green fruit yield and quality of different chilli genotypes in black soil. Int. J. Curr. Res. Biosci. Plant Biol. 3(3), 34-38.

Ranganna, S., 1979. Manual of Analysis of Fruits and Vegetable Products. Tata McGraw Hill Book Company, New Delhi.

\section{How to cite this article:}

Sharma, M., Sharma, A., 2016. Per se performance of chilli genotypes and hybrids across four environments for yield, horticultural and quality traits. Int. J. Curr. Res. Biosci. Plant Biol. 3(9), 51-56. doi: http://dx.doi.org/10.20546/ijcrbp.2016.309.007 